\title{
Determining the available throughput capacity of the railways using simulation for intelligent management of the transportation process
}

\author{
Vadim Shmal ${ }^{1, *}$, Evgenia Prokofieva ${ }^{1}$, and Lily Aysina ${ }^{1}$ \\ ${ }^{1}$ Russian University of Transport (MIIT), $9 \mathrm{~b} 9$ Obrazcova Street, Moscow, 127994, Russia
}

\begin{abstract}
The article is devoted to the problem of determining the available throughput capacity of the railways and assessment of possible infrastructure capacity losses using the simulation method. As a result, the simulation model of the intermediate station has been proposed, which determines the order of trains passing. This model can be used for determination the available throughput capacity in the Throughput Development and Processing Abilities Department and the Train Schedule Department of the Central Directorate of Traffic Management, a branch of JSC «Russian Railways».
\end{abstract}

\section{Introduction}

One of the most important recent problems in railway transport is the lack of throughput and carrying capacity of the railway infrastructure.

The main value of JSC «Russian Railways» for carrying out production tasks are infrastructure resources, which are also the main strategic means for the Company's development.

Today the railroad transport system has changed the structure of the car fleet, the system of planning, normalization and management of car traffic volumes.

In the circumstances, the slightest deviations from the adopted technology lead to unproductive use of existing infrastructure capacities, disruptions in the efficiency and rhythm of the operation of the railways and, as a result, losses in the financial result.

The train schedule (hereinafter referred to as the TS) is the main document ensuring the interaction of all railway services and units for carrying out the transportation process taking into account traffic safety. The main indicator of the effectiveness of the TS is the throughput capacity, calculated according to the current "Instruction for calculating the available railroad capacity" $[1,2,3]$, which contains formulas that do not always give an unambiguous result. To maximize the use of the railway transport infrastructure and the management of the transportation process, it is necessary to know the value of the available throughput capacity and be able to determine the «narrow» places on the railway network.

Given the limited capacity of the infrastructure resources, much attention is paid to technological solutions based on the cross-cutting principles of the organization of the

* Corresponding author: morgoth-lan@yandex.ru 
transportation process and the planning of transportation, taking into account the throughput and processing capabilities of public and non-public infrastructures (consignees, consignors), and the possibility of using extraterritorial approaches of JSC «Russian Railways» divisions interaction on regional and network levels.

\section{Materials and methods}

To obtain the most complete information about the capabilities of a particular railway section or stage, it is necessary to simulate the process of its operation. This approach will allow more accurate determination of the available throughput capacity, which in turn will be able to provide answers to the following two questions:

- whether the railway section will be able to master the forecasted traffic dimensions;

- whether there is a need for modernization of the railway section under consideration.

In the conditions of multivariate organization of the transportation process, the construction of the simulation model will allow «to lose» any variant of the development of events with given initial data.

The ideas of automating the compilation of the TS are described in the literature of the early 20th century. Some scientists developed methods and algorithms that exclude human intervention for scheduling. Others believed that the work of an engineer can not be completely ruled out.

Of course, each method of compiling the TS has certain advantages and disadvantages.

Thus, an automated method can exclude errors and inaccuracies arising from the influence of the «human factor».

On the other hand, the graphist engineer has the ability to improve the graphics from year to year, consistently retaining the best of the previously found layouts, while the machine can not do this yet.

To date, the problem of automating the construction of the TS on the railway network belongs to the class of the most complex problems. An important topic that needs to be solved is the development of a program capable of plotting and evaluating it in the way graphists do today [4].

Currently, both issues (developing a traffic schedule and calculating the available throughput capacity) are resolved separately from each other. The currently functioning engineer-graphist's automated workplace is a software package designed to facilitate the work of the graphist $[5,6]$, which does not allow to fully automate the process. Therefore, the quality of the schedule often depends directly on the qualification of the engineer.

The process of optimization and adjustment of the parameters of the functioning of various aspects of the organization of traffic requires that all possible scenarios of events be taken into account.

To date, there is a large number of software, both foreign and Russian origin. In different degrees, these or other data are taken into account, respectively, the result of the work is also different. Among the foreign developments is worth noting RailSys with the software product RailPlan, Opantimetable, Smone and others. [7, 8, 9]

Among domestic developments, it is possible to distinguish the hardware and software complex (hereinafter - HSC) «Elbrus», developed by VNIIZhT and allowing to determine graphically the maximum possible throughput capacity of the section under given restrictions. Use of simulation models allows you to compare different options and come to an optimal solution. The application of the HSC «Elbrus» already contributes to the implementation of the regulatory TS by making adjustments to the existing work taking into account the infrastructure capabilities, rolling stock features, etc. $[1,10,11]$

The application of simulation modeling finds a positive evaluation in the article «Complex of methods for monitoring train advancement and simulation of transportation 
processes». $[6,11]$ The authors argue that simulation methods open the possibility of a detailed quantitative analysis of the effectiveness of measures for organizing traffic.

\section{Results}

In this article, we propose to use the possibilities of simulation modeling of the work of the railway section. With this approach, the object under study is replaced by a model, with sufficient accuracy, describing the actual system over which experiments are performed. This will automate the calculation of the throughput capacity of the railway section and the development of a schedule of train traffic. There is also an opportunity to fully take into account the track development of stations, the technical equipment of stations and sections, the types of train schedules.

The model of the railway section (Figure 1) consists of separate components: models of railway intermediate stations and models of distances. The models of the stations, in turn, include models of railway station' constrictions and receiving-departure tracks. To coordinate the work of the railway section elements, the model of the train dispatcher is used. In this case, the approach of trains to the section from the technical stations is simulated.

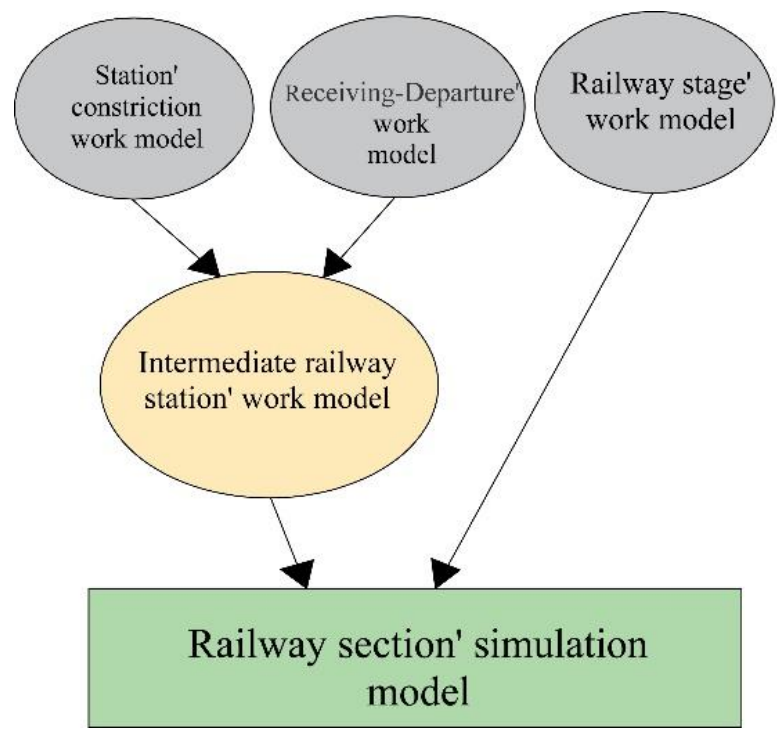

Fig. 1. Diagram of a simulation model of a railway section

In practical application, the simulation model can be compared with the indicators of the hardware-software complex of dispatch control (HSC DC). If the readings do not match, you can provide the dispatcher with notifications for making operative decisions.

The use of discrete-event modeling will determine the cronological sequence of events and the state of the system at any time. Standard components of the system of discrete-event simulation [7] (time, event list, event statistics, termination conditions, system cycles) allow to generate initial data.

Input information:

- time of arrival and departure of the train from the station;

- the minimum time for the train to stay at the station;

- train number;

- train category; 
- the number of the stage track from which the train arrives and to which it leaves.

Output information:

- train number;

- the actual time of arrival and departure of the train;

- the actual time of the train's stay at the station;

- delay time on arrival and departure of the train;

- number of the station track to which the train arrives;

- route of arrival and departure of the train.

Discrete-event modeling allows to obtain as output data the non-productive downtimes of trains and wagons, depending on the accepted technology of organization and management of operational work. Assess the negative consequences of technological violations possible through the calculation of possible losses of infrastructure capacity. Valuation of these losses will help to offset the negative consequences and can be the basis for developing effective management decisions.

The memory of the simulation model is presented in Table 1. It is divided into long-term and short-term. Depending on the category, the memory contains various information.

Table 1. Simulation model' memory structure

\begin{tabular}{|c|c|}
\hline \multicolumn{2}{|c|}{ Simulation model' memory } \\
\hline Long-term memory & Short-term memory \\
\hline The number of stage and station tracks & The schedule of trains' arrival and departure at stations \\
\hline \multirow{2}{*}{$\begin{array}{c}\text { The list of trains' arrival and departure } \\
\text { routes }\end{array}$} & $\begin{array}{c}\text { Railway tracks' occupancy by the trains' receiving, } \\
\text { departure and proceeding }\end{array}$ \\
\cline { 2 - 2 } & The occupancy of trains' receiving and departure routes \\
\hline The station' approach quantity & The train's priorities concerning arrival and departure \\
\hline $\begin{array}{c}\text { The mutual influence connections between } \\
\text { the routes - both - inside the constriction } \\
\text { and - between the constrictions }\end{array}$ & The priorities of trains' receiving and departure routes \\
\cline { 2 - 2 } & The present time point \\
\hline
\end{tabular}

To encode the diagram of the constriction of the station, a hostile route table is used (Fig. 2), which lists all possible routes for receiving and departure trains (in rows and columns). The hostility of the routes is denoted by one, zero - parallelism.

\begin{tabular}{|c|c|c|c|c|c|c|c|c|c|c|}
\hline Route & 1 & 2 & 3 & 4 & 5 & 6 & 7 & 8 & 9 & 10 \\
\hline 1 & 1 & 1 & 1 & 1 & 1 & 1 & 1 & 0 & 0 & 0 \\
\hline 2 & 1 & 1 & 1 & 1 & 1 & 1 & 1 & 0 & 0 & 0 \\
\hline 3 & 1 & 1 & 1 & 1 & 1 & 1 & 1 & 0 & 0 & 0 \\
\hline 4 & 1 & 1 & 1 & 1 & 1 & 1 & 1 & 0 & 0 & 0 \\
\hline 5 & 1 & 1 & 1 & 1 & 1 & 1 & 1 & 1 & 0 & 0 \\
\hline 6 & 1 & 1 & 1 & 1 & 1 & 1 & 1 & 1 & 0 & 0 \\
\hline 7 & 1 & 1 & 1 & 1 & 1 & 1 & 1 & 1 & 1 & 1 \\
\hline 8 & 0 & 0 & 0 & 0 & 1 & 1 & 1 & 1 & 1 & 1 \\
\hline 9 & 0 & 0 & 0 & 0 & 0 & 1 & 1 & 1 & 1 & 1 \\
\hline 10 & 0 & 0 & 0 & 0 & 0 & 0 & 1 & 1 & 1 & 1 \\
\hline
\end{tabular}

Fig.2. An example of hostility table for station routes

It is worth noting that the lack of simulation is the need to input a large amount of input data. It is proposed to automate this process, which does not completely eliminate the above- 
mentioned drawback, but will significantly reduce it. To simplify the introduction of the structure of the pointer constriction, it is proposed to use a designer that allows constructing a mimic diagram of a station with any track development. Then the scheme of the station (Figure 3a) is represented as a directed graph (Fig. 3b).

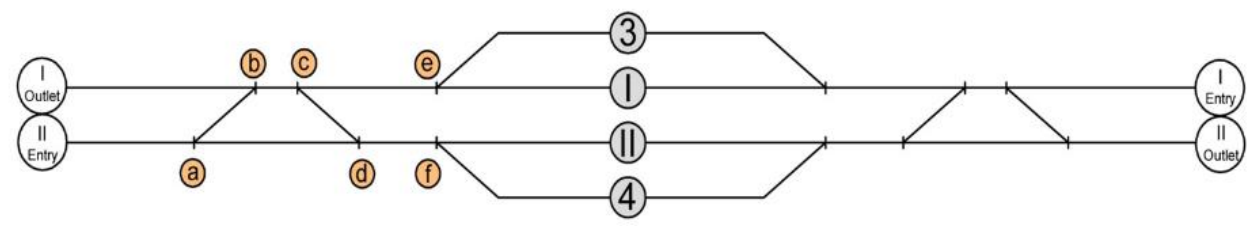

a) non-scale scheme of the station

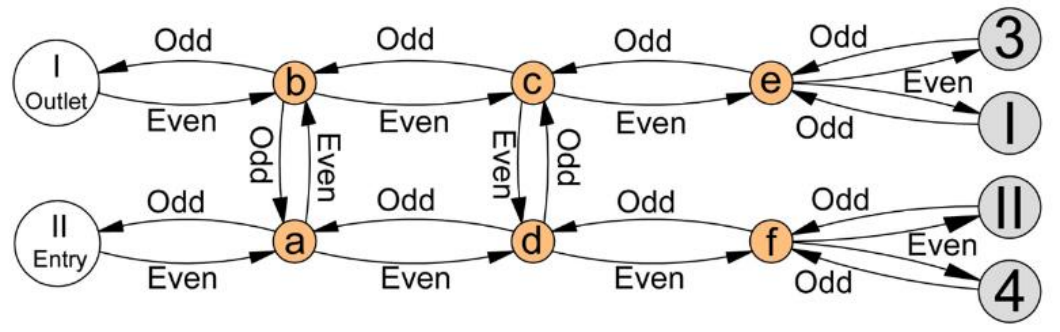

b) station diagram in the form of a directed graph

Fig.3. Definition of station routes

The nodes are stage tracks, switches and station tracks. The possible directions of the motion of even and odd trains are indicated. Thus, the designer makes it possible to choose the optimal route according to the minimum number of traversed nodes.

To determine hostility, routes are considered as a chain of individual elements (Fig. 4). For clarity, as an example, you can bring the principle of the station operator. Compares the elements that are included in a particular route. With the coincidence of at least one link, a conclusion is made about hostility and a table of hostilities is automatically constructed.

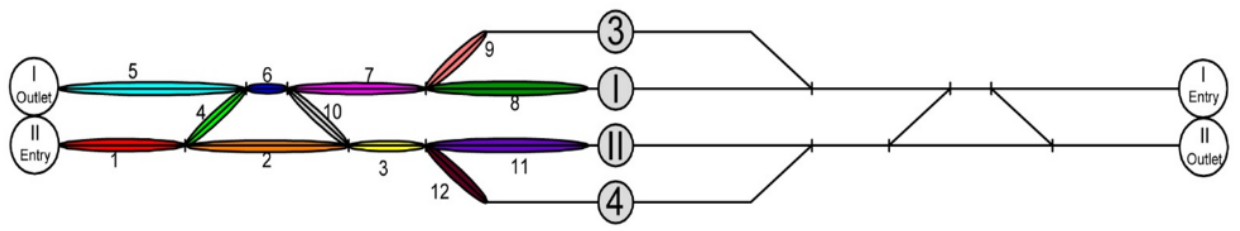

\section{Arrival routes}

$$
\begin{aligned}
& \text { II Entry }->4: 1,2,3,12 \\
& \text { II Entry }->\text { II }: 1,2,3,11 \\
& \text { II Entry -> I : } 1,4,6,7,8 \\
& \text { II Entry }->3: 1,4,6,7,9
\end{aligned}
$$

\section{Departure routes}

$$
\begin{aligned}
& 3 \text {-> I Outlet : } 9,7,6,5 \\
& \text { I -> I Outlet }: 8,7,6,5 \\
& \text { II -> I Outlet : } 11,3,10,6,5 \\
& 4 \text {-> I Outlet : } 12,3,10,6,5
\end{aligned}
$$

Fig.4. Identifying hostile routes 
When entering the input information, the system can automatically determine the time of the occupation of a certain route chain by train of different categories. The number assigned to the train determines its category and priority. The type of locomotive for the section is indicated in the TS, the traction characteristics determine the possible time for the passage of the haul. The speed of moving around the station is also regulated. Modeling the development of events can take place in several iterations. In the event of a conflict situation with the coincidence of one or more input data that complicates the assignment of priority, the system calculates according to the integral scoring system. Expert judgment determines in advance the significance of each parameter. The result of the sum of the products of points assigned to the input value and the value of the expert estimate for this parameter determines the technological evaluation of the variant. Further, an evaluation of alternative options is made. The input data for each situation is compared and the priority is assigned to the train, which has a total of more points. Table 2 shows an example of expert assessment of the importance of parameters.

Table 2. Assignment of the integral weight to the parameter by an expert estimate

\begin{tabular}{|c|c|c|c|c|c|c|c|}
\hline \multirow{2}{*}{ No } & \multirow{2}{*}{ Estimated parameters } & \multirow{5}{*}{ Integral weight } & \multicolumn{5}{|c|}{ Expert assessments } \\
\cline { 3 - 8 } & & E1 & E2 & E3 & E4 & E5 \\
\hline 1 & Arrival accuracy & $\mathbf{0 . 1 9 6}$ & 4 & 3 & 4 & 5 & 4 \\
\hline 2 & Departure accuracy & $\mathbf{0 . 1 8 6}$ & 4 & 3 & 4 & 4 & 4 \\
\hline 3 & Stay duration & $\mathbf{0 . 2 3 5}$ & 5 & 5 & 5 & 4 & 5 \\
\hline 4 & Train category & $\mathbf{0 . 1 5 7}$ & 2 & 4 & 4 & 3 & 3 \\
\hline 5 & Variability of the station route & $\mathbf{0 . 2 2 5}$ & 4 & 5 & 5 & 5 & 4 \\
\hline
\end{tabular}

Table 2 summarizes expert opinions (E1-E5) on parameter estimation. In table 3, for example, trains of various categories are presented in order to determine their priority. Each input value is assigned a certain number of points. The technological evaluation of the variant is the sum of the product of the parameter and its integral weight.

Table 3. Prioritization of the option using technology assessment

\begin{tabular}{|c|c|c|c|c|c|c|c|}
\hline Subpoint \# & \# & 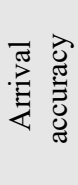 & 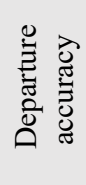 & 点 & 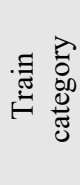 & 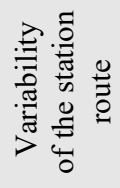 & 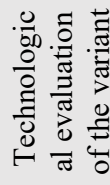 \\
\hline 1 & 7110 & 5 & 5 & 5 & 5 & 5 & 4,9950 \\
\hline 2 & 6316 & 5 & 4 & 5 & 4 & 5 & 4,6520 \\
\hline 3 & 215 & 5 & 4 & 5 & 5 & 5 & 4,8090 \\
\hline 4 & 8517 & 3 & 3 & 3 & 2 & 3 & 2,8400 \\
\hline 5 & 3009 & 2 & 2 & 3 & 3 & 3 & 2,6150 \\
\hline
\end{tabular}

Technological evaluation of the variant allows us to determine the top-down hierarchy of priority. So, the train 7110 has the highest priority, and the train 3009 - the lowest.

The cyclic algorithm of operation of the station model functions simultaneously with the time counter. In the first iteration, the calculation is made of the receipt or departure of the 
claimed train with the highest priority. In subsequent iterations, the possibility of parallel reception or departure of trains is checked. Further, the analysis of the decision results for the estimated time period (day) is performed.

When identifying various violations of technology, the program is trained by adjusting the priorities of trains and routes. In the same way, it is possible to correct the solution at the request of the operator.

\section{Conclusion}

With the use of simulation modeling, it is possible to «lose» the behavior of the station in various situations with a sufficient degree of accuracy and change in the structure of the train traffic, in the implementation of various reconstructive measures, including changes in the technology of operation of the stations, and to assess the resources of the infrastructure.

\section{References}

1. S. A. Vinogradov. Bulletin of the joint academic Council of JSCo «Russian Railways», 3, 32 (2012)

2. Instructions for calculating the available throughput of railways (JSCo «Russian Railways», 2011)

3. Methodology for determining the throughput and carrying capacity of public railway infrastructure (Russian Ministry of transport, 2018)

4. A.T. Osminin, V.A. Anisimov, N.A. Klyuev, L.A. Osminin, V.V. Anisimov. Railway transport, 4, 19 (2012)

5. A.A. Abramov, G.M. Bilenko Modern Automated Transportation Management Systems (Moscow, 2002)

6. I.M. Kokurin, V.L. Belozerov. Izvestiya Petersburg University of Communications, 2, 31 (2012)

7. Yu.O.Pazoisky, V.N. Shmal, I.S. Abdulaev. Proceedings of the International Scientific and Practical Conference «Transport Systems: Development Trends» (2016)

8. S.A. Branshitov, A.M. Shirvanyan, D.A. Tumchenok Information management systems, 6, 73 (2014)

9. RailSys 10. RailSys Information Brochure [On-line]. http://www.rmcon.de (circulation date $05 / 03 / 2018$ )

10. E. Kudryavtseva. VNIIZhT introduces on the railway automated complex «Elbrus» URL: http://www.gudok.ru 11/06/2015 (circulation date April 13, 2013)

11. Information technology: Elbrus URL: http://elbrus-r.ru/ (circulation date 15.02.2018). 